\title{
Singular Solutions and Prolongation of Holomorphic Solutions to Nonlinear Differential Equations
}

\author{
By
}

\section{Takao KOBAYASHI}

\section{\$1. Introduction}

We consider nonlinear differential equations with holomorphic coefficients in the complex domain. They admit interesting singular solutions. First of all we give two concrete examples.

Example 1.1. For any holomorphic function $\varphi(x)$, put $T=t-\varphi(x)$. Then $\mathrm{KdV}$ equation

$$
u_{t t t}-6 u u_{t}+u_{x}=0 \quad(i, x \in \mathbb{C})
$$

has a family of solutions which are singular on $T=0$ :

$$
u=2 T^{-2}-\frac{1}{6} \varphi_{x}+g T^{2}-\frac{1}{36} \varphi_{x x} T^{3}+h T^{4}-\frac{1}{24} g_{x} T^{5}+\cdots,
$$

where $g=g(x)$ and $h=h(x)$ are arbitrary holomorphic functions.

Example 1.2. The equation

$$
u_{t t t}+4 t u u_{t t}+u u_{x x}+x u=0 \quad(t, x \in \mathbf{C})
$$

has a solution which is singular on $t=0$ :

$$
u=t^{-2}-\frac{x}{24} t+\frac{x^{2}}{2304} t^{4}-\frac{1}{188928} t^{5}+\frac{x^{3}}{926208} t^{7}+\cdots .
$$

The Laurent series (1.1b) and (1.2b) are not formal ones, but do converge to define exact solutions (see Theorem 3.1). On the other hand linear differential equations, since the surface $t-\varphi(x)=0$ or $t=0$ is noncharacteristic, never admit such singular ones (see [15]).

Communicated by T. Kawai, April 16, 1997. Revised July 7, 1997.

1991 Mathematics Subject Classification (s) : 35A20

* Department of Mathematics. Science University of Tokyo, Noda, Chiba, 278-8510, Japan takao@ma.noda.sut.ac.jp 
Formal series such as (1.1b) are used by Weiss [13], [14] et al. But their point of view is to study the algebraic structure, for example to obtain Bäcklund transform, which is called Painleve analysis, and they were not concerned with the convergence of the series itself.

Ishii [4] studied singular solutions to general nonlinear partial differential equations and showed first the convergence under certain conditions. But the above two examples do not satisfy them: in Example 1.1, arbitrary functions $g$ and $h$ are not allowed, and in Example 1.2, the exponent by his definition is not -2 but -1 and we can show that there exist no singular solutions with exponent -1 (see Theorem 4.7).

Motivated by this, after changing the coordinates suitably if necessary, we consider nonlinear differential equations which may be written in the form:

$$
\partial_{t}^{m} u=f\left(t, x ;\left(\partial_{t}^{\prime} \partial_{x}^{\alpha} u\right)_{\substack{j \leq m-1 \\ j+|\alpha| \leq m}}\right) \quad\left(t \in \mathbb{C}, x \in \mathbb{C}^{d}\right) .
$$

We define an exponent to (1.3) with respect to $t=0$ (see Definition 2.5) $(1$ ) construct singular solutions with this exponent (Theorem 3.1), and (11) show that there exist no singular solutions whose singularities on $t=0$ are weaker than this exponent (Theorem 4.7).

We finally remark that Leichtnam [7], Nabaji-Wagschal [9]. Nahaji [8] constructed solutions which are singular on a characteristic surface.

\section{Acknowledgement}

The author would like to thank Professor Sunao Ōuchi for informing him that the convergence of formal solutions follows also from the estimates given in [10]. This work was completed while he stayed at University of Paris VI. He is grateful for their hospitality.

\section{§2. Notations and Characteristic Exponemt}

We use $\mathbb{N}$ for the nonnegative integers, while $\mathbb{N}_{>0}$ denotes the positive integers. The variable in $\mathbb{C}$ is denoted by $t$ and $x=\left(x_{1}, \ldots, x_{d}\right)$ in $\mathbb{C}^{d}$ with $d \in \mathbb{N}$ $\left(d=0\right.$ means we consider an ordinary differential equation). We write $\partial_{t}=\partial / \partial t$ and $\partial_{x_{t}}=\partial / \partial x_{\imath}$, and use the standard multi-index notation: if $\alpha=\left(\alpha_{1}, \ldots, \alpha_{d}\right) \in$ $\mathbb{N}^{d}$, we set

$$
\begin{gathered}
|\alpha|:=\alpha_{1}+\cdots+\alpha_{d}, \quad \alpha !:=\alpha_{1} ! \cdots \alpha_{d} ! \\
x^{\alpha}:=x_{1}^{\alpha_{1} \cdots x_{d}^{\alpha_{d}},} \quad \partial_{x}^{\alpha}:=\partial_{x_{1}}^{\alpha_{1} \cdots \partial_{x_{d}}^{\alpha_{d}},} \\
\alpha \geq \beta \quad \text { if } \quad \alpha_{\imath} \geq \beta_{\imath} \text { for } 1 \leq i \leq d .
\end{gathered}
$$

For $m \in \mathbb{N}_{>0}$, we define a set of multi-indices $\Lambda$ by

$$
\Lambda:=\left\{(j, \alpha) \in \mathbb{N} \times \mathbb{N}^{d}: j<m, j+|\alpha| \leq m\right\},
$$

and for $\imath=u(t, x)$, we put 


$$
\partial^{\Lambda} u:=\left(\partial_{t}^{\prime} \partial_{x}^{\alpha} u\right)(\jmath, \alpha) \in \Lambda
$$

The variable corresponding to $\partial_{t}^{\prime} \partial_{x}^{\alpha} u$ is denoted by $Z_{j, \alpha}$ and the totality of $Z_{j, \alpha}$ 's by $Z$. It is convenient to extend the multi-index notation to variables with suffix $(j, \alpha) \in \Lambda$ : if $\mu=\left(\mu_{j, \alpha}\right)_{(j, \alpha) \in \Lambda} \in \mathbb{N}^{\# \Lambda}$, we set

$$
\begin{aligned}
& |\mu|:=\sum_{(\jmath, \alpha) \in \Lambda} \mu_{\jmath, \alpha .} \quad \mu !:=\prod_{(,, \alpha) \in \Lambda} \mu_{,, \alpha ! .} \\
& Z^{\mu}:=\prod_{(\jmath, \alpha) \in \Lambda}\left(Z_{j, \alpha}\right)^{\mu_{, \alpha}} . \quad \partial_{Z}^{\mu}:=\prod_{(\jmath, \alpha) \in \Lambda}\left(\partial_{Z_{j \alpha}}\right)^{\mu_{r \alpha}}, \\
& \left(\partial^{\Lambda} \imath\right)^{\mu}:=\prod_{(\jmath, \alpha) \in \Lambda}\left(\partial_{f}^{\prime} \partial_{x}^{\alpha} \imath\right)^{\mu, \alpha} . \\
& \mu \geq \nu \quad \text { if } \quad \mu_{\jmath, \alpha} \geq \nu_{,, \alpha} \text { for all }(j, \alpha) \in \Lambda \text {. }
\end{aligned}
$$

We study nonlinear differential equations of the form

$$
\partial_{t}^{m} u=f\left(t, x ; \partial^{\Lambda} u\right) .
$$

locally near the origin in $\mathbb{C} \times \mathbb{C}^{d}$. We assume $f(t, x ; Z)$ is holomorphic in $\Omega \times$ $\mathbb{C}^{\# \Lambda}$, where $\Omega$ is an open neighborhood of the origin in $\mathbb{C} \times \mathbb{C}^{d}$. We may write

$$
f(t, x ; Z)=\sum_{\mu \in \mathscr{U}} f_{\mu}(t, x) Z^{\mu},
$$

with a subset $M \subset \mathbb{N}^{\# \Lambda}$ and $f_{\mu} \in \mathscr{O}(\Omega)$, where $\mathscr{O}(\Omega)$ is the space of holomorphic functions in $\Omega$. For simplicity, we exclude $\mu$ from $\mathcal{M}$ if $f_{\mu} \equiv 0$. We expand $f_{\mu}$ in $t$ and denote by $k_{\mu}(\in \mathbb{N})$ the vanishing order of $f_{\mu}$ on $t=0$, namely,

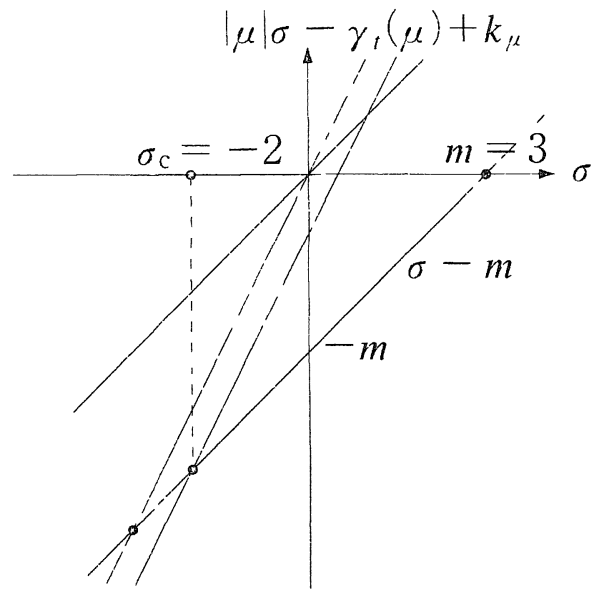

Figure 1. Characteristic exponent of Example 1.2 


$$
f_{\mu}(t, x)=t^{k_{u}} \sum_{k=0}^{\infty} f_{\mu, k}(x) t^{k}, \quad \text { with } \quad f_{\mu, 0}(x) \not \equiv 0 .
$$

For each $\mu \in M$, we write $\gamma_{t}(\mu)$ for the total number of the differentiations with respect to $t$ in $\left(\partial^{\Lambda} u\right)^{\mu}$. i.e.,

$$
\gamma_{t}(\mu):=\sum_{(j, \alpha) \in \Lambda} j \cdot \mu_{j, \alpha} \circ
$$

Now we define a characteristic exponent, which plays an important role in the following sections.

Definition 2.1. The characteristic exponent $\sigma_{c}$ of (2.1) with respect to the surface $t=0$ is

$$
\sigma_{c}:=\sup _{\substack{\mu \in M \\|\mu| \geq 2}} \frac{\gamma_{t}(\mu)-m-k_{\mu}}{|\mu|-1} .
$$

We assign $u$ weight $\sigma, \partial_{t}$ weight -1 and $t$ weight 1 , then the total weight of the term $f_{\mu}\left(\partial^{\Lambda} u\right)^{\mu}$ is $|\mu| \sigma-\gamma_{t}(\mu)+k_{\mu}$. Draw lines defined by $\sigma \mapsto\left(\sigma,|\mu| \sigma-\gamma_{t}(\mu)\right.$ $\left.+k_{\mu}\right)$. Then the term in the right hand side of (2.5) is the coordinate of the intersection with the line $\sigma-m$, which is the weight of the left hand side of (2.1) (see Figure 1).

Example 2.2. In Example 1.1 we have

$$
m=3, \quad \gamma_{t}(\mu)=1, \quad|\mu|=2, \quad k_{\mu}=0 \quad \text { and } \quad \sigma_{c}=-2,
$$

where $\mu$ corresponds to the term $u u_{t}$.

Although the characteristic exponent of (1.1a) does not depend on the surfaces, in general it depends on them.

Example 2.3. The characteristic exponent of $(1.2 \mathrm{a})$ is -2 with respect to $t=0$ and is -1 with respect to $t-a x=0$ if $a \neq 0$. In fact, if we change $t \rightarrow t-$ $a x,(1.2 \mathrm{a})$ becomes

$$
u_{t t t}+\left(4(t+a x)+a^{2}\right) u u_{t t}+u\left(u_{x x}-2 a u_{t x}\right)+x u=0,
$$

whose exponent is -1 , and $(1.2 \mathrm{a})$ has the following solution:

$$
u=\frac{3}{a(a+4 x)}(t-a x)^{-1}-\frac{48 x}{a^{2}(a+4 x)^{3}}-\frac{48\left(a^{2}+4 a x-16 x^{2}\right)}{a^{3}(a+4 x)^{5}}(t-a x)+\cdots,
$$

which is singular on $t-a x=0$ with exponent -1 .

Proposition 2.4. The characteristic exponent $\sigma_{c}$ is invariant with respect to coordinate changes which keep the variable $t$.

Proof. Let $s=t, y=y(t, x)$ be such a coordinate change. Since $\partial_{t}=\partial_{s}+$ 
$\sum_{k} a_{k} \partial_{y_{k}}$ and $\partial_{x_{i}}=\sum_{k} b_{t, k} \partial_{y_{k}}$, where $a_{k}, b_{i, k}$ are holomorphic near the origin, we have

$$
\left(\partial_{t}^{j} \partial_{x}^{\alpha} u\right)^{\mu_{, \alpha} \alpha}=\left(\sum_{\substack{l \leq j \\ l+|\beta| \leq j+|\alpha|}} c_{l, \beta}^{j, \alpha} \cdot \partial_{s}^{l} \partial_{y}^{\beta} u\right)^{\mu_{, \alpha}}
$$

with some holomorphic functions $c_{l, \beta}^{j, \alpha}$. Notice that the total number of differentiation with respect to $s$ of each nonlinear term in the right hand side of (2.7) is less than or equal to $j \cdot \mu_{j, \alpha}$. Therefore we can write

$$
\left(\partial^{\Lambda} u\right)^{\mu}=\sum_{\substack{\left.|\nu|=|\mu| \\ \gamma_{\ell}(\nu) \leq \gamma_{t}^{\prime} \mu\right)}} a_{\mu, \nu} \cdot\left(\partial_{s, y}^{\Lambda} u\right)^{\nu},
$$

with some holomorphic functions $a_{\mu, \nu}$. Because the vanishing order $k_{\mu}$ of $f_{\mu}$ is invariant with this coordinate change, the one $\widetilde{k}_{\nu}$ of $f_{\mu} \cdot a_{\mu, \nu}$ is greater than or equal to $k_{\mu}$. These imply that

$$
\frac{\gamma_{t}(\nu)-m-\widetilde{k}_{\nu}}{|\nu|-1} \leq \frac{\gamma_{t}(\mu)-m-k_{\mu}}{|\mu|-1},
$$

where the left hand side is the exponent of the nonlinear term $f_{\mu} \cdot a_{\mu, \nu}\left(\partial_{s, y}^{A} u\right)^{\nu}$. Taking the supremum on all $\mu, \nu$ such that $\mu \in M,|\nu|=|\mu|, \gamma_{t}(\nu) \leq \gamma_{t}(\mu)$, we obtain that the characteristic exponent with respect to the new coordinate system is less than or equal to the original one.

By exchanging the role of coordinate systems we obtain the reverse inequality. So the two characteristic exponents are equal.

Lemma 2.5. If $f\left(t, u ; \partial^{A} u\right)$ includes only terms of which the order of differentiation with respect to $t$ are less than or equal to $m_{0}(\leq m-1)$, then $\sigma_{c} \leq m_{0}$. Especially it always holds $\sigma_{c} \leq m-1$.

Proof. By the assumption and the definition (2.4), we have $\gamma_{t}(\mu) \leq m_{0}|\mu|$. Hence

$$
\frac{\gamma_{t}(\mu)-m-k_{\mu}}{|\mu|-1} \leq m_{0}-\frac{\left(m-m_{0}\right)+k_{\mu}}{|\mu|-1}<m_{0}
$$

Remark. The above proof shows that $\sigma_{c}$ is strictly less than $m_{0}$ if $\mathcal{M}$ is a finite set.

In the following sections, $m_{0}(\leq m-1)$ denotes the maximum order of differentiation with respect to $t$ in $f\left(t, x ; \partial^{\Lambda} u\right)$.

\section{§3. Construction of Singular Solutions}

3.1. Formal solution. In this section we construct a solution which is singular on $t=0$ to the equation

$$
\partial_{t}^{m} u=f\left(t, x ; \partial^{\Lambda} u\right)
$$


To do this, we need to assume that

(A-1) $f(t, x ; Z)=\sum_{\mu \in M} f_{\mu}(t, x) Z^{\mu}$ is a polynomial in $Z$, that is $M$ is a finite set, and is of degree greater than or equal to 2, i.e., (3.1) is not a linear equation.

Since $\mathcal{M}$ is a finite set, the characteristic exponent

$$
\sigma_{c}=\max _{\substack{\mu \in \mathcal{M} \\|\mu| \geq 2}} \frac{\gamma_{t}(\mu)-m-k_{\mu}}{|\mu|-1}
$$

is a rational number strictly less than $m_{0}$, and the subset $\mathcal{M}^{*}$ of $\mathcal{M}$ defined by

$$
\mathscr{M}^{*}:=\left\{\mu \in M:|\mu| \sigma_{c}-\gamma_{t}(\mu)+k_{\mu}=\sigma_{c}-m\right\}
$$

is not empty. We call the nonlinear term corresponding to $\mu$ in $\mathcal{M}^{*}$ principal nonlinear term. The second assumption is

(A-2) If $\mu \in M^{*}$ then $\mu_{, \alpha}=0$ for $|\alpha| \geq 1$, namelv. all principal nonlinear terms do not contain the derivatives with respect to $x$.

Remark. The condition (A-2) seems somewhat strong. However if (A-2) does not hold, we replace the surface $t=0$ with another one. For example, the equation

$$
u_{t t}+u u_{x}+u_{x}=0 \quad(t, x \in \mathbb{C}) .
$$

whose characteristic exponent is -2 , does not satisfy $(A-2)$. But if we make a coordinate change, $(t, x) \rightarrow(t-a x, x)$ with $a \neq 0$, the above equation becomes

$$
u_{t t}-a u u_{t}+u u_{x}+u_{x}-a u_{t}=0 .
$$

This equation satisfies $(A-2)$, because the characteristic exponent is -1 and the principal nonlinear term is $a u u_{t}$. In fact, we have the following solutions to (3.4a) for $a \neq 0$ :

$$
u=-\frac{2}{a}(t-a x)^{-1}-1+g \cdot(t-a x)^{1}+\frac{g_{x}}{2 a}(t-a x)^{2}+\cdots,
$$

where $g=g(x)$ is an arbitrary holomorphic function.

Now for $\rho \in \mathbb{R}$ and $j \in \mathbb{N}$, we set

$$
[\rho ; j]:=\rho(\rho-1) \cdots(\rho-j+1)=\frac{\Gamma(\rho+1)}{\Gamma(\rho-j+1)} \quad([\rho ; 0]:=1),
$$

and introduce two polynomials which depend only on principal nonlinear terms:

$$
\begin{aligned}
P_{c}(x ; \eta) & :=\left[\sigma_{c} ; m\right]-\sum_{\mu \in \mu^{*}} f_{\mu, 0}(x)\left(\prod_{(,, \alpha) \in \Lambda}\left[\sigma_{c} ; j\right]^{\mu_{j} \alpha}\right) \eta^{|\mu|-1} \\
& =\left[\sigma_{c}: m\right]-\sum_{\mu \in \mu^{*}} f_{\mu, 0}(x)\left(\prod_{j=0}^{m-1}\left[\sigma_{c} ; j\right]^{\mu, 0}\right) \eta^{|\mu|-1} \quad \text { by }(\mathrm{A}-2) .
\end{aligned}
$$


(3.7) $Q_{c}(x: \eta: \rho)$

$$
\begin{aligned}
:=\left[\rho+\sigma_{c} ; m\right]-\sum_{\mu \in \mathcal{M}^{*}} f_{\mu, 0}(x)\left(\prod_{(, \alpha) \in \Lambda}\left[\sigma_{c} ; j\right]^{\mu_{, \alpha}}\right)\left(\sum_{(,, \alpha) \in \Lambda} \mu_{,, \alpha} \frac{\left[\rho+\sigma_{c} ; j\right]}{\left[\sigma_{c} ; j\right]}\right) \eta^{|\mu|-1} \\
=\left[\rho+\sigma_{c} ; m\right]-\sum_{\mu \in M^{+}} f_{\mu, 0}(x) \cdot\left(\sum_{j=0}^{m-1} \mu_{\jmath, 0}\left[\rho+\sigma_{c} ; j\right]\left[\sigma_{c} ; 0\right]^{\mu_{1,0} \ldots}\right. \\
\left.\cdots\left[\sigma_{c} ; j\right]^{\mu, 0-1} \cdots\left[\sigma_{c} ; m-1\right]^{\mu m-1,0}\right) \eta^{|\mu|-1} \quad \text { by }(\mathrm{A}-2) .
\end{aligned}
$$

Note that $P_{c}$ and $Q_{c}$ are of degree $\max _{\mu \in \mu^{*}}|\mu|-1$ in $\eta$ and that $Q_{c}$ is also a polynomial in $\rho$ of degree $m$.

We construct a solution to (3.1) in the form:

$$
u(t, x):=t^{\sigma c} \sum_{n=0}^{\infty} u_{n}(x) t^{n / p},
$$

where $p \in \mathbb{N}_{>0}$ is the denominator of the reduced fraction $\sigma_{c}$, $u_{n}$ 's are holomorphic in a common neighborhood of the origin of $\mathbb{C}^{d}$ and $u_{0} \neq \equiv$.

Substitute (3.8) into (3.1) and equate the coefficient of $t^{\sigma_{c-m+n} / p}$ to 0 for each $n \in \mathbb{N}$, and we obtain the following recurrence equations:

$$
\begin{cases}P_{c}\left(x ; u_{0}\right) \cdot u_{0}=0 & (n=0), \\ Q_{c}\left(x ; u_{0} ; \frac{n}{p}\right) \cdot u_{n}=R_{n}\left(x ; \ldots, \partial_{x}^{\alpha} u_{0}, \ldots, \partial_{x}^{\alpha} u_{n-1}\right)|\alpha| \leq m & (n=1,2, \ldots) .\end{cases}
$$

Here $R_{n}$ is a polynomial in and depends only on $u_{0}, \ldots, u_{n-1}$ and their derivatives. Let us assume the following:

(A-3) The equation $P_{c}(x ; \eta)=0$ in $\eta$ has at least one solution $\eta=u u_{0}(x)$ which is holomorphic in a neighborhood of $x=0$ and $u_{0}(x) \neq \equiv$.

(A-4) One of the following holds for each $n=1,2,3, \ldots$ :

(a) $Q_{c}\left(0 ; u_{0}(0): \frac{n}{p}\right) \neq 0$,

(b) $Q_{c}\left(x ; \imath_{0}(x) ; \frac{n}{p}\right) \equiv 0$ and $R_{n}\left(x ; \ldots, \partial_{x}^{\alpha} u_{0}, \ldots, \partial_{x}^{\alpha} u_{n-1}\right) \equiv 0$,

(c) $Q_{c}\left(0 ; u_{0}(0) ; \frac{n}{p}\right)=0, Q_{c}\left(x ; u_{0}(x) ; \frac{n}{p}\right) \not \equiv 0$ and

$$
Q_{c}\left(x ; u_{0}(x) ; \frac{\eta}{p}\right) \text { divides } R_{n}\left(x ; \ldots, \partial_{x}^{\alpha} u_{0}, \ldots, \partial_{x}^{\alpha} u_{n-1}\right) .
$$

Remark. In case of (a) or (c), $u_{n}$ is determined uniquely, and in case of (b). $u_{n}(x)$ may be any holomorphic function.

Note also that since $Q_{c}(x ; \eta ; \rho)$ is also a polynomial in $\rho$ of degree $m$, the equation 


$$
Q_{c}\left(0 ; u_{0}(0) ; \rho\right)=0
$$

has at most $m$ distinct roots.

Theorem 3.1. Suppose (A-1), (A-2), (A-3) and (A-4) are satisfied. Then we can construct a solution to (3.1) of the form:

$$
u=t^{\sigma_{c}} \sum_{n=0}^{\infty} u_{n}(x) t^{n / p}
$$

where $u_{n}^{\prime}$ s are holomorphic in a common neighborhood of the origin in $\mathbb{C}_{x}^{d}$ and $u_{0} \equiv 0$. Moreover all formal solutions of the above form converge near the origin in $\mathbb{C}_{t} \times \mathbb{C}_{x}^{d}$.

Example 3.2. KdV equation (1.1a) has

$$
\sigma_{c}=-2, P_{c}(x ; \eta)=-24+12 \eta, \quad Q_{x}(x ; \eta=2 ; \rho)=(\rho+1)(\rho-4)(\rho-6) \text {. }
$$

In this example, $Q_{c}(x ; 2 ; n)$ vanishes identically for $n=4,6$, and $\left.R_{4}\right|_{u_{0}=2}$ and $\left.R_{6}\right|_{u_{0}=2}$ also vanish identically. Therefore $u_{4}$ and $u_{6}$ may be any holomorphic functions.

Example 3.3. Equation $(1.2 \mathrm{a})$ has

$$
\sigma_{c}=-2, \quad P_{c}(x ; \eta)=-24+24 \eta, \quad Q_{c}(x ; \eta=1 ; \rho)=\rho(\rho-2)(\rho-3)+24 .
$$

In this example, $Q_{c}(x ; 1 ; n) \neq 0$ for all $n \in \mathbb{N}_{>0}$ and $u_{n}$ 's are determined uniquely. On the other hand, Equation (2.6a), which comes from (1.2a) after a coordinate change, has

$$
\begin{gathered}
\sigma_{c}=-1, \quad P_{c}(x ; \eta)=-6+2 a(a+4 x) \eta, \\
Q_{c}\left(x ; \eta=\frac{3}{a(a+4 x)} ; \rho\right)=(\rho+1)\left(\rho^{2}-4 \rho+6\right) .
\end{gathered}
$$

and $\imath_{n}$ 's are determined uniquely.

Example 3.4. Equation (3.4b) has

$$
\sigma_{c}=-1, \quad P_{c}(x ; \eta)=2+a \eta, \quad Q_{c}\left(x ; \eta=-\frac{2}{a} ; \rho\right)=(\rho+1)(\rho-2) .
$$

In this example, $Q_{c}\left(x ;-\frac{2}{a} ; n\right)$ vanishes identically for $n=2$, and $\left.R_{2}\right|_{u_{0}=-\frac{2}{a}}$ also vanish identically. Therefore $u_{2}$ may be any holomorphic function.

3.2. Convergence of formal spolution. By the assumptions (A-3) and (A-4) the existence of a formal solution is trivial, so we need only to show the convergence of (3.10).

We will use the following result by Gerard and Tahara (see [2], [3, Chapter 8]): Consider the following nonlinear differential equation:

$$
\left(t \partial_{t}\right)^{m} w=F\left(t, x ;\left(\left(t \partial_{t}\right)^{j} \partial_{x}^{\alpha} w\right)_{(j, \alpha) \in \Lambda}\right)
$$


where $F(t, x ; Z)$ is holomorphic in a neighborhood of $(t, x ; Z)=(0,0 ; 0)$ and satisfies

$$
\begin{aligned}
& F(0, x ; 0) \equiv 0, \\
& \frac{\partial F}{\partial Z_{j, \alpha}}(0, x ; 0) \equiv 0 \quad \text { if }|\alpha|>0 .
\end{aligned}
$$

The characteristic polynomial of $(3.11)$ is

$$
C(p, x):=\rho^{m}-\sum_{j=0}^{m-1} \frac{\partial F}{\partial Z_{j, 0}}(0, x ; 0) \rho^{j}
$$

Theorem 3.5 (Gérard-Tahara). If $C(n, 0) \neq 0$ for all $n \in \mathbb{N}_{>0}$, then (3.11) has a unique formal solution $w=\sum_{n=1}^{\infty} w_{n}(x) t^{n}$ with $w(0, x) \equiv 0$, where $w_{n}(x)$ are holomorphic on a common neighborhood of the origin in $\mathbb{C}^{d}$. Moreover this power series is convergent and holomorphic near the origin in $\mathbf{C}_{t} \times \mathbf{C}_{x}^{d}$.

For $N \in \mathbb{N}_{>0}$, we set

$$
w_{N}(t, x):=\sum_{n=0}^{\infty} u_{N+n+1}(x) t^{\frac{n+1}{p}}
$$

Proposition 3.6. If the formal series (3.10) satisfies the equation (3.1), then $w_{N}$ satisfies the following differential equation:

$$
Q_{c}\left(x ; u_{0}(x) ; t \partial_{t}+\frac{N}{p}\right) w_{N}=t^{1 / p} \cdot G\left(t^{1 / p}, x:\left(\left(t \partial_{t}\right)^{\jmath} \partial_{x}^{\alpha} w_{N}\right)_{(\jmath, \alpha) \in \Lambda}\right),
$$

where $Q_{c}$ is a differential operator of order $m$ oblained from (3.7) by replacing (3.5) with $j$-th order ones

$$
\left[t \partial_{t}+\rho ; j\right]=\left(t \partial_{t}+\rho\right)\left(t \partial_{t}+\rho-1\right) \cdots\left(t \partial_{t}+\rho-j+1\right),
$$

and $G(\tau, x ; Z)$ is a polynomial in $Z$ with coefficients holomorphic near the origin in $\mathbb{C}_{\tau, x}^{d+1}$.

Proof. Put

$$
a_{N}(t, x):=t^{\sigma c} \sum_{n=0}^{N} u_{n}(x) t^{n / p}, \quad b_{N}(t, x):=t^{\sigma c} \sum_{n=N+1}^{\infty} u_{n}(x) t^{n / p},
$$

then $u=a_{N}+b_{N}$ and $b_{N}=t^{\sigma_{c}+\frac{N}{b}} w_{N}$. Using the identity

$$
\partial_{t}^{j}\left(t^{\rho} w\right)=t^{\rho-j} \cdot\left(\left[t \partial_{t}+\rho ; j\right] w\right) \quad \text { for all } j \in \mathbf{N} \text { and } \rho \in \mathbf{R},
$$

we obtain

$$
\begin{aligned}
\left(\partial^{\Lambda} a_{N}\right)^{\mu} & =\prod_{(j, \alpha) \in \Lambda}\left(\partial_{t}^{j} \partial_{x}^{\alpha}\left(t^{\sigma_{c}}\left(u_{0}+t^{\frac{1}{p}} u_{1}+\cdots+t^{\frac{N}{p}} u_{N}\right)\right)\right)^{\mu_{, \alpha}} \\
& =t^{|\mu| \sigma_{c}-\gamma t(\mu)}\left[\prod_{(, \alpha) \in \Lambda}\left(\left[\sigma_{c} ; j\right] \partial_{x}^{\alpha} u_{0}\right)^{\mu_{,, \alpha}}+t^{\frac{1}{p}} \mathbf{O}\left(t^{\frac{1}{p}}, x\right)\right],
\end{aligned}
$$


with some function $\mathbb{O}(\tau, x)$ holomorphic near $(\tau, x)=(0,0)$, and

$$
\begin{aligned}
\left(\partial^{\Lambda} b_{N}\right)^{\nu} & =\prod_{(j, \alpha) \in \Lambda}\left(\partial_{t}^{j} \partial_{x}^{\alpha}\left(t^{\sigma_{c}+\frac{N}{p}} w_{N}\right)\right)^{\nu_{\alpha} \alpha} \\
& =t^{|\nu| \sigma_{c}-\gamma_{t}(\nu)+|\nu| \frac{N}{p}} \prod_{(1, \alpha) \in \Lambda}\left(\left[t \partial_{t}+\sigma_{c}+\frac{N}{p} ; j\right] \partial_{x}^{\alpha} w_{N}\right)^{\nu_{, \alpha}} .
\end{aligned}
$$

By (3.18), (3.19), (2.3) and by the identity $\gamma_{t}(\mu-\nu)=\gamma_{t}(\mu)-\gamma_{t}(\nu)$, we have

$$
\begin{aligned}
& f_{\mu} \cdot\left(\partial^{\Lambda} a_{N}\right)^{\mu-\nu}\left(\partial^{\Lambda} b_{N}\right)^{\nu}=t^{|\mu| \sigma_{c}-\gamma_{t}(\mu)+k_{\mu}+|\nu| \frac{N}{D}} \\
& \cdot\left[\prod_{(j, \alpha) \in A} f_{\mu, 0}\left(\left[\sigma_{c} ; j\right] \partial_{x}^{\alpha} u_{0}\right)^{\mu, \alpha-\nu, \alpha}\left(\left[t \partial_{t}+\sigma_{c}+\frac{N}{p} ; j\right] \partial_{x}^{\alpha} w_{N}\right)^{\nu_{, \alpha}}\right. \\
& \left.+t^{\frac{1}{p}} \mathbb{P}\left(t^{\frac{1}{p}}, x ;\left(\left(t \partial_{t}\right)^{\prime} \partial_{x}^{\alpha} w_{N}\right)_{(, \alpha) \in \Lambda}\right)\right] .
\end{aligned}
$$

where $\mathbb{P}(\tau, x ; Z)$ is a polynomial in $Z$ whose coefficients are holomorphic near $(\tau, x)=(0,0)$. In the following we will use the same $\mathbb{O}(\tau, x)$ or $\mathbb{P}(\tau, x ; Z)$ to represent some functions as above.

Substituting $u=a_{N}+b_{N}$ into (3.1) yields

$$
\begin{aligned}
& \partial_{t}^{m} u-f\left(t . x ; \partial^{\Lambda} u\right) \\
& =\partial_{t}^{m} a_{N}+\partial_{t}^{m} b_{N}-\sum_{\mu \in \mathscr{U}} \sum_{0 \leq \nu \leq \mu} f_{\mu} \cdot\left(\begin{array}{l}
\mu \\
\nu
\end{array}\right)\left(\partial^{\Lambda} a_{N}\right)^{\mu-\nu}\left(\partial^{\Lambda} b_{N}\right)^{\nu} \\
& =\partial_{t}^{\mathrm{m}} a_{N}-\sum_{\mu \in . M} \sum_{\nu \leq \mu} \cdots+t^{\Sigma_{c}-m+\frac{N}{p}}\left[t \partial_{t}+\sigma_{c}+\frac{N}{p} ; m\right] w_{N}-\sum_{\mu \in M} \sum_{\nu \leq \mu} \cdots \\
& \text { (I) } \\
& \text { (II) }
\end{aligned}
$$

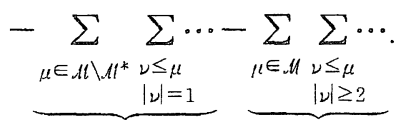

Since $u_{n}$ 's satisfy the recurrence equations (3.9), the first term is

$$
\text { ( I ) }=\partial_{t}^{\mathrm{m}} a_{N}-f\left(t, x ; \partial^{\Lambda} a_{N}\right)=t^{\sigma_{c}-m+\frac{N}{p}+\frac{1}{p}} \mathbb{O}\left(t^{\frac{1}{p}}, x\right) .
$$

The definition (3.2) of $\sigma_{c}$ implies

$$
|\mu| \sigma_{c}-\gamma_{t}(\mu)+k_{\mu}=\sigma_{c}-m \quad \text { if } \mu \in M^{*} \text {. }
$$

$$
|\mu| \sigma_{c}-\gamma_{t}(\mu)+k_{\mu} \geq \sigma_{c}-m+\frac{1}{p} \quad \text { if } \mu \in \mathcal{M} \backslash \mathcal{M}^{*}
$$

By (3.23). (3.20) and the definition (3.7), the second term is

$$
(\mathbb{I})=t^{\sigma_{c}-n+\frac{N}{p}}\left[Q_{c}\left(x, u_{0} ; t \partial_{t}+\frac{N}{p}\right) w_{N}+t^{\frac{1}{p}} \mathbb{P}\left(t^{\frac{1}{p}}, x:\left(\left(t \partial_{t}\right)^{\jmath} \partial_{x}^{\alpha} w_{N}\right)_{(\jmath, \alpha) \in \Lambda}\right)\right] .
$$


By (3.24), the third one becomes

$$
(\text { III })=t^{\sigma_{c}-m+\frac{N}{p}+\frac{1}{p}} \mathbb{P}\left(t^{\frac{1}{p}}, x ;\left(\left(t \partial_{t}\right)^{\jmath} \partial_{x}^{\alpha} w_{N}\right)_{(\jmath, \alpha) \in \Lambda}\right) .
$$

and by $|\nu| \geq 2$, the fourth one

$$
(\mathbb{N})=t^{\sigma_{c}-n+\frac{2 N}{p}} \mathbb{P}\left(t^{\frac{1}{p}}, x ;\left(\left(t \partial_{t}\right)^{\jmath} \partial_{x}^{\alpha} w_{N}\right)_{(j, \alpha) \in \Lambda}\right) .
$$

Substituting (3.22), (3.25), (3.26) and (3.27) into (3.21), we obtain the desired equation (3.16).

Let us put $\tau=t^{1 / p}$ and

$$
\widetilde{w}_{N}(\tau, x)=\sum_{n=0}^{\infty} \iota_{N+n+1}(x) \tau^{n+1}
$$

Then $\widetilde{w}_{N}(0, x) \equiv 0$, and by substituting the relation $t \partial_{t}=\frac{1}{p} \tau \partial_{\tau}$ to $(3.16), \widetilde{w}_{N}$ satisfies the following differential cquation:

$$
Q_{c}\left(x ; u_{0}(x) ; \frac{1}{p} \tau \partial_{\tau}+\frac{N}{p}\right) \widetilde{w}_{N}=\tau \cdot G \cdot\left(\tau, x ;\left(\left(\frac{1}{p} \tau \partial_{\tau}\right)^{\jmath} \partial_{x}^{\alpha} \widetilde{w}_{N}\right)_{(\jmath, \alpha) \in \Lambda}\right) .
$$

Obviously the above equation satisfies the conditions (3.12) and (3.13), and its characteristic polynomial is

$$
C(\rho, x)=Q_{c}\left(x ; u_{0}(x) ; \frac{1}{p}(\rho+N)\right) .
$$

Therefore if we take $N$ sufficiently large, $C(n, 0) \neq 0$ for all $n \in \mathbb{N}_{>0}$. Now applying Theorem 3.5 to Equation $(3.29)$, we obtain that $\widetilde{w}_{N}$ converges near $(\tau, x)=(0,0)$. This completes the proof of Theorem 3.1 .

\section{\$4. Prolongation of Holomorphic Solutions}

In this section we show that if $u$ is holomorphic in

$$
\Omega_{-}=\{(t, x) \in \Omega: \operatorname{Re} t<0\}
$$

and satisfies Equation (2.1) and if $u$ satisfies a certain boundedness condition which is closely related to the characteristic exponent $\sigma_{c}$, then $u$ is holomorphic near the origin.

When $\sigma_{c}$ is positive, by a change of unknown function $w=\imath-h$, where $h$ is some holomorphic function, the characteristic exponent of the new equation for $w$ may be greater than the original one and we may obtain a singular solution of the form:

$$
u=\text { holomorphic function }+ \text { singular solution by Theorem } 3.1
$$

(see Example 4.2). So we need to define a modified version of characteristic exponent. 
Definition 4.1. For (2.1), we define $\sigma_{c}^{*}$ by

$$
\sigma_{c}^{*}=\sup _{\substack{\mu \in M \\ \nu \leq \mu,|\nu| \geq 2}} \gamma_{t}(\nu)-m-k_{\mu}
$$

Example 4.2. The equation

$$
u_{t t}+6 u u_{t}^{3}+x u_{t}^{2}+u u_{x}=0
$$

has $\sigma_{c}=\frac{1}{3}$ and $\sigma_{c}^{*}=\frac{1}{2}$, and

$$
P_{c}(x ; \eta)=\frac{2}{9}\left(\eta^{3}-1\right), \quad Q_{c}\left(x ; \eta=1, \omega \text { or } \omega^{2} ; \frac{n}{3}\right)=\frac{1}{9}(n+2)(n+3) .
$$

So, for example, putting $u_{0}=1$, we obtain the following solution with exponent $\frac{1}{3}$ :

$$
u=t^{1 / 3}-\frac{x}{12} t^{2 / 3}+\frac{x^{2}}{240} t^{3 / 3}+\frac{x^{3}}{5184} t^{4 / 3}-\frac{277 x^{4}}{5443200} t^{5 / 3}+\cdots .
$$

On the other hand, if we replace the unknown variable $u$ with $w+\frac{1}{3} g^{2}$, where $g$ $=g(x)$ is an arbitrary holomorphic function with $g(0) \neq 0$, then $(4.2 \mathrm{a})$ becomes

$$
w_{t t}+2 g^{2} w_{t}^{3}+6 w w_{t}^{3}+x w_{t}^{2}+\left(w+\frac{1}{3} g^{2}\right)\left(w_{x}+\frac{2}{3} g g_{x}\right)=0
$$

which has

$$
\sigma_{c}=\frac{1}{2}, \quad P_{c}(x ; \eta)=\frac{1}{4}\left(g^{2} \eta^{2}-1\right), \quad Q_{c}\left(x ; \eta= \pm \frac{1}{g} ; \frac{n}{2}\right)=\frac{1}{4}(n+1)(n+2) .
$$

Therefore $(4.2 \mathrm{a})$ has the following singular solutions with exponent $\frac{1}{2}$ :

(4.2c) $u=\frac{1}{3} g^{2}+\frac{1}{g} t^{1 / 2}+\left(-\frac{1}{2 g^{4}}-\frac{x}{6 g^{2}}\right) t^{2 / 2}+\left(\frac{5}{8 g^{7}}+\frac{7 x}{24 g^{5}}+\frac{x^{2}}{36 g^{3}}\right) t^{3 / 2}+\cdots$.

Lemma 4.3. The characteristic exponents $\sigma_{c}$ and $\sigma_{c}^{*}$ enjoy the following:

(1) $\sigma_{c} \leq \sigma_{c}^{*} \leq m_{0}(\leq m-1)$.

(i1) If $\sigma_{c} \leq 0$, then $\sigma_{c}=\sigma_{c}^{*}$.

Proof. (i) The first inequality is trivial. The second one is the same as that of Lemma 2.5.

(ii) It always holds

$$
\gamma_{t}(\nu)-m-k_{\mu} \leq \gamma_{t}(\mu)-m-k_{\mu} \quad \text { for } \nu \leq \mu,
$$

and if $\sigma_{c} \leq 0$,

$$
\gamma_{t}(\mu)-m-k_{\mu} \leq 0 \quad \text { for all } \mu \in M
$$

These inequalities imply for all $\mu \in M$ and $\nu \leq \mu$ with $|\nu| \geq 2$ 


$$
\frac{\gamma_{t}(\nu)-m-k_{\mu}}{|\nu|-1} \leq \frac{\gamma_{t}(\nu)-m-k_{\mu}}{|\mu|-1} \leq \frac{\gamma_{t}(\mu)-m-k_{\mu}}{|\mu|-1} \leq \sigma_{c} .
$$

This shows $\sigma_{c}^{*} \leq \sigma_{c}$.

Definition 4.4. For $\sigma \in \mathbb{R}$, we define $\delta_{c}(\sigma)$ by

$$
\begin{aligned}
\delta_{c}(\sigma): & =\inf _{\substack{\mu \in . \mu \\
\nu \leq \mu,|\nu| \geq 2}}(|\nu|-1) \sigma-\gamma_{t}(\nu)+m+k_{\mu} \\
& =\inf _{\substack{\mu \in \cdot \mu \\
\nu \leq \mu,|\nu| \geq 2}}\left(|\nu| \sigma-\gamma_{t}(\nu)+k_{\mu}\right)-(\sigma-m) .
\end{aligned}
$$

Lemma 4.5. $\delta_{c}(\cdot)$ enjoys the following properties:

(1) $\delta_{c}(\sigma) \geq 0$ if and only if $\sigma \geq \sigma_{c}^{*}$.

(ii) If $\sigma>\sigma_{c}^{*}$, then $\delta_{c}(\sigma)>0$.

(iii) $\delta_{c}\left(m_{0}\right)>0$.

(1v) If $\delta_{c}(\sigma)>0$ and $\sigma \leq m_{0}$, then there is a constant $\delta>0$ such that

$$
|\nu| \sigma-\gamma_{t}(\nu)+k_{\mu} \geq \sigma-m+\delta \text { for all } \mu \in \mathcal{M}, \nu \leq \mu .
$$

Proof. (1) is clear, because $\delta_{c}(\sigma) \geq 0$ means

$$
(|\nu|-1) \sigma \geq \gamma_{t}(\nu)-m-k_{\mu} \text { for all } \mu \in \mathcal{M}, \nu \leq \mu,|\nu| \geq 2 \text {. }
$$

(11) Note that for $|\nu| \geq 2$

$$
(|\nu|-1) \sigma-\gamma_{t}(\nu)+m+k_{\mu} \geq(|\nu|-1) \sigma_{c}^{*}-\gamma_{t}(\nu)+m+k_{\mu}+\left(\sigma-\sigma_{c}^{*}\right) .
$$

This implies $\delta_{c}(\sigma) \geq \sigma-\sigma_{c}^{*}>0$.

(111) follows from

$$
(|\nu|-1) m_{0}-r_{t}(\nu)+m+k_{\mu} \geq(|\nu|-1) m_{0}-|\nu| m_{0}+m+k_{\mu} \geq m-m_{0} .
$$

Here we have used the inequality $\gamma_{t}(\nu) \leq|\nu| m_{0}$.

(iv) It is enough to show (4.4) for $|\nu|=0,1$. For $|\nu|=0,(4.4)$ follows directly from $\sigma \leq m-1$, and for $|\nu|=1$, it follows from $\gamma_{t}(\nu) \leq m_{0}$.

Definition 4.6. Let $u$ be holomorphic in $\Omega_{-}$and $\sigma \in \mathbf{R}$. We say that $u$ is bounded of order $\sigma$ if there is a constant $M>0$ such that the following estimates hold for $(t, x) \in \Omega_{-}$:

$$
|u(t, x)| \leq M \mid \operatorname{Re} t^{\sigma} \quad \text { if } \sigma \leq 0
$$

or

$$
\left|\partial_{t}^{\prime} \iota(t, x)\right| \leq\left\{\begin{array}{ll}
M & \text { for } j=0,1, \ldots,\lfloor\sigma\rfloor, \\
M|\operatorname{Re} t|^{\sigma-j} & \text { for } j=\lfloor\sigma\rfloor+1,
\end{array} \quad \text { if } \sigma>0 .\right.
$$

Here $\lfloor\sigma\rfloor$ is the greatest integer less than or equal to $\sigma$. 
For example, $t^{\sigma} \cdot h(t, x)$ with a holomorphic function $h(t, x)$ is bounded of order $\sigma$, and $\log t \cdot h(t, x)$ is bounded of order $-\varepsilon$ for any $\varepsilon>0$.

Remark. If $u$ is bounded of order $\sigma$ in $\Omega$. then by Cauchy's inequality and by shrinking $\Omega_{-}$if necessary, we may assume that for $(t, x) \in \Omega_{-}$

$$
\left|\partial_{t} u(t, x)\right| \leq M \mid \operatorname{Re} t^{\sigma-\jmath} \quad \begin{cases}\text { for } j=0,1, \ldots, m-1 & \text { if } \sigma \leq 0, \\ \text { for } j=\lfloor\sigma\rfloor+1, \ldots, m-1 & \text { if } \sigma>0 .\end{cases}
$$

Note that if $u$ is bounded of order $\sigma$, it is also bounded of order $\widetilde{\sigma}$ for all $\tilde{\sigma} \leq \sigma$. Now we can state the second main theorem.

Theorem 4.7. Suppose that $u$ is holomorphic and bounded of order $\sigma$ in $\Omega$ and that $u$ satisfies Equation (2.1) in $\Omega_{\text {-. If }} \delta_{c}(\sigma)>0$, then $u$ is holomorphic in a neighborhood of the origin in $\mathbb{C} \times \mathbb{C}^{d}$. Especially if $\sigma>\sigma_{c}^{*}$ or $\sigma=m_{0}$, then $u$ is holomorphic near the origin in $\mathbb{C} \times \mathbb{C}^{d}$.

Corollary 4.8. If $u \in \mathscr{O}\left(\Omega_{-}\right)$satisfies Equation (2.1) and the derivatives of $u$ $u p$ to order $m_{0}$ are bounded in $\Omega_{-}$, then $u$ is holomorphic near the origin in $\mathbb{C}_{t} \times \mathbb{C}_{x}^{d}$.

Example 4.9. Examples 1.1, 1.2 and 2.3 give singular solutions which are bounded of order $\sigma_{c}=\sigma_{c}^{*}$, and Example 4.2 gives ones of order $\sigma_{c}^{*}$ with $\sigma_{c}^{*}>\sigma_{c}$. On the other hand, for the equation

$$
u_{t}=\exp (-u)
$$

we have $\sigma_{c}=\sigma_{c}^{*}=0$ and $\delta_{c}(0)=1>0$. This equation has a solution $u=\log t$. which is bounded of order $-\varepsilon$ for any $\varepsilon>0$. However Theorem 4.7 implies that this equation has no solutions of bounded of order $\sigma_{c}^{*}=0$.

\section{$\S 5$. Proof of Prolongation Theorem}

We use the method of majorants. For two formal power series centered at $x=0$

$$
g=\sum_{\beta} g_{\beta} \cdot x^{\beta}, \quad G=\sum_{\beta} G_{\beta} \cdot x^{\beta},
$$

we write $g \ll G$ or $G \gg g$ if $\left|g_{\beta}\right| \leq G_{\beta}$ for all $\beta \in \mathbb{N}^{d}$. For two formal ones centered at $(t, x)=(-\varepsilon, 0)$

$$
g=\sum_{k, \beta} g_{k, \beta} \cdot(t+\varepsilon)^{k} x^{\beta}, \quad G=\sum_{k, \beta} G_{k, \beta} \cdot(t+\varepsilon)^{k} x^{\beta},
$$

we write $g \ll_{\varepsilon} G$ or $G \gg_{\varepsilon} g$ if $\left|g_{k, \beta}\right| \leq G_{k, \beta}$ for all $(k, \beta) \in \mathbb{N} \times \mathbb{N}^{d}$.

The following auxiliary functions are very useful to study nonlinear differential equations (cf. [6], [12]). For $i=0,1, \ldots, m$, we put 


$$
\theta^{(i)}(z):=\sum_{n=0}^{\infty} \frac{z^{n}}{(n+1)^{i+2}} \quad(z \in \mathbf{C})
$$

Lemma 5.1 ( $\mathrm{Lax})$. There exists positive constant $C_{1}>1$ such that

$$
\left(\theta^{(\imath)}(z)\right)^{2} \ll C_{1} \theta^{(\imath)}(z) \quad i=0.1, \ldots, m
$$

Proof. It follows from

$$
\begin{aligned}
\sum_{k=0}^{n}\left(\frac{n+1}{(k+1)(n-k+1)}\right)^{l} & \leq 2 \sum_{k=0}^{\lfloor n / 2\rfloor}\left(\frac{n+1}{(k+1)(n-k+1)}\right)^{l} \\
& =2 \sum_{k=0}^{\lfloor n / 2\rfloor} \frac{1}{(k+1)^{l}}\left(\frac{n+1}{n-k+1}\right)^{l} \leq 2^{l+1} \sum_{k=0}^{\infty} \frac{1}{(k+1)^{l}} .
\end{aligned}
$$

With this $C_{1}$ we define

$$
\varphi^{(1)}(z):=C_{1}^{-1} \theta^{(1)}(z) \quad(i=0,1, \ldots, m),
$$

and for $\varepsilon>0, \rho \gg 1$ and $R>0$

$$
\begin{gathered}
\varphi_{R}^{(2)}(x):=\varphi^{(2)}\left(\frac{1}{R}\left(x_{1}+\cdots+x_{d}\right)\right) . \\
\varphi_{\rho, R, \varepsilon}^{(1)}(t, x):=\varphi^{(1)}\left(\frac{1}{R}\left(\rho(t+\varepsilon)+x_{1}+\cdots+x_{d}\right)\right) .
\end{gathered}
$$

Lemma 5.2. (1) For all $n \in \mathbb{N}_{>0}$ the following majorant incqualities hold:

$$
\begin{gathered}
\left(\varphi^{(1)}(z)\right)^{n} \ll \varphi^{(1)}(z) . \\
\left(\varphi_{R}^{(l)}(x)\right)^{n} \ll \varphi_{R}^{(z)}(x) . \\
\left(\varphi_{\rho, R, \varepsilon}^{(t)}(t, x)\right)^{n} \ll_{\varepsilon} \varphi_{\rho, R, \varepsilon}^{(z)}(t, x) .
\end{gathered}
$$

(11) There exists a constant $C_{2}>0$ such that for $i=1, \ldots, m$

$$
C_{2} \varphi^{(1-1)}(z) \ll \frac{d}{d z} \varphi^{(2)}(z) \ll \varphi^{(2-1)}(z) .
$$

$$
C_{2} \varphi_{R}^{(1-1)}(x) \ll R \frac{\partial}{\partial x_{k}} \varphi_{R}^{(t)}(x) \ll \varphi_{R}^{(1-1)}(x) .
$$

$$
C_{2} \varphi_{\rho, R, \varepsilon}^{(t-1)}(t, x) \ll_{\varepsilon} R \frac{\partial}{\partial x_{k}} \varphi_{\rho, R, \varepsilon}^{(l)}(t, x) \ll_{\varepsilon} \varphi_{\rho, R, \varepsilon}^{(2-1)}(t, x)
$$$$
C_{2} \varphi_{\rho, R, \varepsilon}^{(1-1)}(t, x) \ll_{\varepsilon} \frac{R}{\rho} \frac{\partial}{\partial t} \varphi_{\rho, R, \varepsilon}^{(1)}(t, x) \ll_{\varepsilon} \varphi_{\rho, R, \varepsilon}^{(2-1)}(t, x) .
$$ 
(iii) The following series of majorant inequalities hold:

$$
\begin{gathered}
\varphi^{(m)}(z) \ll \varphi^{(m-1)}(z) \ll \cdots \ll \varphi^{(1)}(z) \ll \varphi^{(0)}(z), \\
\varphi_{R}^{(m)}(x) \ll \varphi_{R}^{(m-1)}(x) \ll \cdots \ll \varphi_{R}^{(1)}(x) \ll \varphi_{R}^{(0)}(x), \\
\varphi_{\rho, R, \varepsilon}^{(m)}(t, x) \ll_{\varepsilon} \varphi_{\rho, R, \varepsilon}^{(m-1)}(t, x) \ll_{\varepsilon} \cdots \ll_{\varepsilon} \varphi_{\rho, R, \varepsilon}^{(1)}(t, x) \ll_{\varepsilon} \varphi_{\rho, R, \varepsilon}^{(0)}(t, x) .
\end{gathered}
$$

Proof. (1) follows directly from Lemma 5.1.

(11) It is enough to prove $(5.6 \mathrm{a})$, which holds with $C_{2}=\left(\frac{1}{2}\right)^{m+2}$, because

$$
\left(\frac{1}{2}\right)^{1+2} \leq\left(\frac{n+1}{n+2}\right)^{\imath+2}<1 \quad \text { for all } n \in \mathbb{N}
$$

(iii) is trivial by the definition.

We use the following polydiscs.

$$
\begin{gathered}
\Delta(R ;-\varepsilon):=\left\{(t, x) \in \mathbb{C} \times \mathbb{C}^{d}:|t+\varepsilon|<R,\left|x_{i}\right|<R, i=1,2, \ldots, m\right\}, \\
\Delta(R):=\left\{(t, x) \in \mathbb{C} \times \mathbb{C}^{d}:|t|<R,\left|x_{i}\right|<R, i=1,2, \ldots, m\right\}, \\
\Delta^{\prime}(R):=\left\{x \in \mathbb{C}^{d}:\left|x_{i}\right|<R, i=1,2, \ldots, m\right\} .
\end{gathered}
$$

Lemma 5.3. There is a constant $C_{3}>0$ such that if $g(t, x) \in \mathcal{O}(\Delta(2 R ;-\varepsilon))$ and $|g(t, x)| \leq M$, or $h(x) \in \mathscr{O}\left(\Delta^{\prime}(2 R)\right)$ and $\mid h(x) ! \leq M$, then for $i=0,1,2, \ldots, m$, we have

$$
g(t, x) \ll_{\varepsilon} C_{3} M \varphi_{\rho, R, \varepsilon}^{(\imath)}(t, x) \quad \text { for all } \rho \geq 1,
$$

$$
h(x) \ll C_{3} M \varphi_{R}^{(2)}(x) .
$$

Here $C_{3}$ is independent of $R, \rho$ and $\varepsilon$.

Proof. Cauchy's inequality implies

$$
g \ll_{\varepsilon} \frac{M}{1-\frac{1}{2 R}\left((t+\varepsilon)+x_{1}+\cdots+x_{d}\right)} .
$$

On the other hand, we have

$$
\frac{1}{1-\frac{z}{2 R}}=\sum_{n} \frac{1}{2^{n}}\left(\frac{z}{R}\right)^{n} \ll c_{i} \sum_{n} \frac{1}{(n+1)^{i+2}}\left(\frac{z}{R}\right)^{n}=c_{i} C_{1} \varphi^{(i)}\left(\frac{z}{R}\right)
$$

with $c_{\imath}=\sup _{n} \frac{(n+1)^{1+2}}{2^{n}}$. Therefore (5.8) holds with $C_{3}=C_{1} \max \left\{c_{0}, \ldots, c_{m}\right\}$.

Lemma 5.4. There is a constant $C_{4}>1$ such that

$$
t \cdot \varphi_{\rho, R, \varepsilon}^{(0)}(t, x) \ll_{\varepsilon} C_{4}\left(\varepsilon+4 \frac{R}{\rho}\right) \varphi_{\rho, R, \varepsilon}^{(0)}(t, x) .
$$

Here $C_{4}$ is independent of $\rho, R$ and $\varepsilon$. 
Proof. Since

$$
t=(-\varepsilon)+(t+\varepsilon) \ll_{\varepsilon} \varepsilon+\frac{R}{\rho}\left(\frac{1}{R}\left(\rho(t+\varepsilon)+x_{1}+\cdots+x_{d}\right)\right),
$$

and

$$
\varphi_{\rho, R, \varepsilon}^{(0)}(t, x)=C_{1}^{-1}\left(1+\frac{1}{4 R}\left(\rho(t+\varepsilon)+x_{1}+\cdots+x_{d}\right)+\cdots\right),
$$

(5.9) holds with $C_{4}=C_{1}$.

Let us fix $R_{0}>0$ with $\Delta\left(2 R_{0}\right) \Subset \Omega$ and $0<R<R_{0}$ with

$$
0<R \leq \min \left\{1, \frac{2}{9} C_{4}^{-1} R_{0}\right\} .
$$

We have assumed that $f(t, x ; Z)$ is holomorphic in $\Omega \times \mathbb{C}^{\# A}$, so for each $L>0$, there is a constant $A_{L}>0$ such that

$$
|f(t, x ; Z)| \leq A_{L} \quad \text { for }(t, x) \in \Delta\left(2 R_{0}\right), \text { and }\left|Z_{j, \alpha}\right| \leq L \quad((j, \alpha) \in \Lambda) .
$$

Lemma 5.5. There is a constant $C_{5}>0$ which is independent of $L$ and $\rho$ such that for each $L>0, \rho \geq 1$ and $\varepsilon=\frac{R}{2 \rho}$, we have

$$
f_{\mu}(t, x) \ll_{\varepsilon} C_{5} A_{L} L^{-|\mu|} \rho^{-k_{\mu}} \varphi_{\rho, R, \varepsilon}^{(0)}(t, x) .
$$

Proof. Appling Cauchy's inequality to (2.2) and (2.3) with (5.11) yields

$$
\left|f_{\mu}(t, x)\right| \leq A_{L} L^{-|\mu|} \quad \text { in } \Delta\left(2 R_{0}\right),
$$

and

$$
\left|f_{\mu, k}(x)\right| \leq A_{L} L^{-|\mu|}\left(2 R_{0}\right)^{-\left(k_{\mu}+k\right)} \quad \text { in } \Delta^{\prime}\left(2 R_{0}\right) .
$$

By Lemma 5.3, we have

$$
\begin{aligned}
f_{\mu, k}(x) & \ll C_{3} A_{L} L^{-|\mu|}\left(2 R_{0}\right)^{-\left(k_{u}+k\right)} \varphi_{R}^{(0)}(x) \\
& \ll_{\varepsilon} C_{3} A_{L} L^{-|\mu|}\left(2 R_{0}\right)^{-\left(k_{u}+k\right)} \varphi_{\rho, R, \varepsilon}^{(0)}(t, x),
\end{aligned}
$$

and by Lemma 5.4

$$
\begin{aligned}
t^{k_{\mu}+k} f_{\mu, k}(x) & \ll_{\varepsilon} C_{3} A_{L} L^{-|\mu|} C_{4}^{k_{a}+k}\left(\varepsilon+4 \frac{R}{\rho}\right)^{k_{u}+k}\left(2 R_{0}\right)^{-\left(k_{u}+k\right)} \varphi_{\rho, R, \varepsilon}^{(0)}(t, x) \\
& \ll_{\varepsilon} C_{3} A_{L} L^{-|\mu|}\left(\frac{9 C_{4} R}{4 \rho R_{0}}\right)^{k_{u}+k} \varphi_{\rho, R, \varepsilon}^{(0)}(t, x) \quad\left(\text { by } \varepsilon=\frac{R}{2 \rho}\right) \\
& \left.\ll_{\varepsilon} C_{3} A_{L} L^{-|\mu|}(2 \rho)^{-\left(k_{u}+k\right)} \varphi_{\rho, R, \varepsilon}^{(0)}(t, x) \quad \text { (by }(5.10)\right) .
\end{aligned}
$$

Substitute the above inequality into (2.3) and we obtain

$$
f_{\mu}(t, x) \ll_{\varepsilon} C_{3} A_{L} L^{-|\mu|}(2 \rho)^{-k \mu} \sum_{k=0}^{\infty}\left(\frac{1}{2 \rho}\right)^{k} \varphi_{\rho, R, \varepsilon}^{(0)}(t, x)
$$




$$
\ll_{\varepsilon} 2 C_{3} A_{L} L^{-|\mu|} \rho^{-k_{\mu}} \varphi_{\rho, R, \varepsilon}^{(0)}(t, x) .
$$

This shows that the assertion holds with $C_{5}=2 C_{3}$.

We have assumed that $u \in \mathscr{O}\left(\Omega_{-}\right)$is bounded of order $\sigma$ with $\delta_{c}(\sigma)>0$. We divide the proof into two cases, $\sigma<0$ and $\sigma \geq 0$.

5.1. Ir case of $\sigma<0$. We consider the following majorant differential equation to $(2.1)$ :

$$
\begin{aligned}
& \partial_{t}^{m} U \gg_{\varepsilon} \sum_{u \in \in_{\mu}} F_{\mu}(t, x)\left(\partial^{\Lambda} U\right)^{\mu} . \\
& \partial_{t}^{j} U(-\varepsilon, x) \gg_{t}^{t} u(-\varepsilon, x) \quad \text { for } j=0.1,2, \ldots, m-1 .
\end{aligned}
$$

Here

$$
F_{\mu}(t, x):=C_{5} A_{L} L^{-|\mu|} \rho^{-k_{\mu}} \varphi_{\rho, R, \varepsilon}^{(0)}(t, x)
$$

is the right hand side of $(5.12)$ and $\varepsilon=\frac{R}{2 \rho}$. If $U$ satisfies the majorant equation (5.13a) with (5.13b), then we have $u \ll_{s} U$. We take $L^{i}$ in the form:

$$
L_{i}^{r}:=K \rho^{-\sigma} R^{m} \varphi_{\rho, R, \varepsilon}^{(m)}(t, x) .
$$

First we consider the initial condition. Since $u$ is bounded of order $\sigma(<0)$, we may take a constant $M>0$ such that for all $0<\varepsilon \ll 1$

$$
\left|\partial_{t}^{\prime} u(-\varepsilon, x)\right| \leq M \varepsilon^{\sigma-1} \quad \text { for } x \in \Delta^{\prime}\left(2 R_{0}\right), \quad j=0,1,2, \ldots, m-1 \text {. }
$$

Hemma 5.6. There is a constant $K_{0}>0$ such that $L$ defined by (5.15) with $K \geq K_{0} M$ satisfies the initial condition (5.13b). Here $K_{0}$ is independent of $\rho \geq 1$.

Proof. By Lemma 5.3 and (5.16)

$$
\partial_{t}^{j} u(-\varepsilon, x) \ll C_{3} M \varepsilon^{\sigma-\jmath} \varphi_{R}^{(m-\jmath)}(x) .
$$

On the other hand, by applying (5.6d) $j$-times

$$
\partial_{t}^{\prime} U \gg \gg_{\varepsilon} C_{2}^{\prime} K \rho^{j-\sigma} R^{m-\jmath} \varphi_{\rho, R, \varepsilon}^{(m-\jmath)}(t, x),
$$

which implies

$$
\partial_{f}^{\jmath} U(-\varepsilon, x) \gg C_{2}^{\jmath} K \rho^{\jmath-\sigma} R^{m-\jmath} \varphi_{R}^{(m-\jmath)}(x) .
$$

for $\left.\varphi_{o, R, \varepsilon}^{(2)}(t, x)\right|_{t=-\varepsilon}=\varphi_{R}^{(z)}(x)$. Therefore (5.13b) holds if

$$
C_{2}^{j} K \rho^{\jmath-\sigma} R^{m-\jmath} \geq C_{3} M \varepsilon^{\sigma-\jmath}=C_{3} M R^{\sigma-\jmath}(2 \rho)^{\jmath-\sigma},
$$

that is, it is enough to take

$$
K_{0}=\max _{0 \leq j \leq m-1} C_{3} C_{2}^{-j} 2^{\jmath-\sigma} R^{\sigma-m}
$$

Next we consider the majorant equation (5.13a). 
Lemma 5.7. Let us take $L>K$. Then there is a constant $\rho_{0}=\rho_{0}(L) \geq 1$ such that $U$ defined by (5.15) with $\rho \geq \rho_{0}$ satisfies the majorant equation (5.13a).

Proof. By (5.6c), (5.6d), we have for all $(j . \alpha) \in \Lambda$

$$
\begin{aligned}
\partial_{t}^{\prime} \partial_{x}^{\alpha} U & \ll_{\varepsilon} K \rho^{j-\sigma} R^{m-\jmath-|\alpha|} \varphi_{\rho, R, \varepsilon}^{(m-|\alpha|)}(t, x) \\
& \ll_{\varepsilon} K \rho^{j-\sigma} \varphi_{\rho, R, \varepsilon}^{(0)}(t, x) \quad(\text { by }(5.10) \text { and } j+|\alpha| \leq m) .
\end{aligned}
$$

and

$$
\left(\partial^{\Lambda} U\right)^{\prime \prime} \ll_{\varepsilon} K^{|\mu|} \rho^{\gamma}{ }^{(\mu)-|\mu| \sigma} \varphi_{\rho, R, \varepsilon}^{(0)}(t, x) .
$$

Substituting the above inequality and (5.14) into the right hand side of (5.13a). we obtain

$$
\text { Right hand side of }(5.13 \mathrm{a}) \ll_{\varepsilon} C_{5} H_{L} \sum_{\mu \in \mathcal{M}} K^{|\mu|} L^{-|\mu|} \rho^{\gamma_{t}(\mu)-|\mu| \sigma-k_{\mu}} \varphi_{\rho, R, \varepsilon}^{(0)}(t, x) \text {. }
$$

Since $\delta_{c}(\sigma)>0$, by Lemma 4.5. we can take $\delta>0$ so that

$$
|\mu| \sigma-\gamma_{t}(\mu)+k_{\mu} \geq \sigma-m+\delta \quad \text { for all } \mu \in . M
$$

which implies

$$
\rho^{\gamma t(\mu)-|\mu| \sigma-k_{u}} \leq \rho^{m-\sigma-\delta}
$$

Using this inequality, we obtain

$$
\text { Right hand side of }(5.13 \mathrm{a}) \ll_{\varepsilon} C_{5} A_{L} \rho^{m-\sigma-\delta} \sum_{\mu \in \mathcal{U}} K^{|\mu|} L^{-|\mu|} \varphi_{\rho, R, \varepsilon}^{(0)}(t, x) .
$$

On the other hand, by $(5.6 \mathrm{~d})$, it holds

$$
\partial_{t}^{m} L^{\tau} \gg_{\varepsilon} C_{2}^{m} K \rho^{m-\sigma} \varphi_{\rho, R, \varepsilon}^{(0)}(t, x) .
$$

Therefore the assertion is valid if

$$
C_{2}^{m} K \rho^{m-\sigma} \geq C_{5} A_{L} \rho^{m-\sigma-\delta} \sum_{\mu \in \mathscr{K}} K^{|\mu|} L^{-|\mu|},
$$

that is,

$$
\rho^{\delta} \geq C_{2}^{-n} C_{5} K^{-1} A_{L} \sum_{\| \in \mathscr{M}} K^{|\mu|} L^{-|\mu|}
$$

where the summation is convergent, since $0<K<L$.

Now take $K \geq K_{0} M, L>K$ and then $\rho \geq \rho_{0}(L)$ and $\varepsilon=\frac{R}{2 \rho}$. Then, by the above two lemma, $U$ defined by (5.15) satisfies the majorant equation $(5.13 \mathrm{a})$ and the initial condition (5.13b), and $L^{\top}$ converges in a domain $\left\{(t, x): \rho|t+\varepsilon|+\left|x_{1}\right|+\cdots\right.$ $\left.+\left|x_{d}\right|<R\right\}$, which contains the origin of $\mathbb{C} \times \mathbb{C}^{d}$ for $\rho \cdot|0+\varepsilon|+0+\cdots+0=\frac{R}{2}<R$. Since $u \ll_{\varepsilon} U, u$ is also holomorphic near the origin of $\mathbf{C} \times \mathbf{C}^{d}$. This complete the proof in case of $\sigma<0$.

5.2. In case $\boldsymbol{\sigma} \geq \mathbf{0}$. Since we have always $\delta_{c}(m-1)>0$, we may assume $\sigma$ 
$\leq m-1$. For sufficiently small $\varepsilon$, we put

$$
v(t, x):=\sum_{j=0}^{\lfloor\sigma\rfloor} \frac{1}{j !} \partial_{i}^{j} u(-\varepsilon, x)(t+\varepsilon)^{j},
$$

and $w=u-v$. Then $w$ satisfies the following equation:

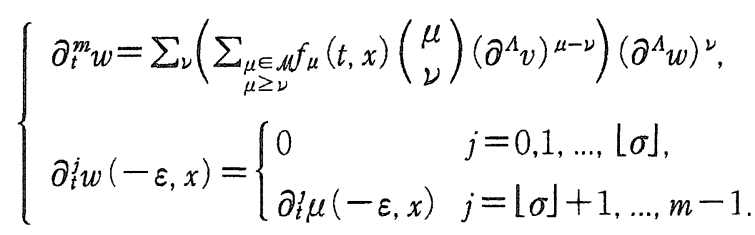

For $u$ is bounded of order $\sigma$, we can take $M_{1}>0$ so that for all sufficiently small $\varepsilon$ and $x \in \Delta^{\prime}\left(2 R_{0}\right)$ we have

$$
\left|\partial_{t}^{j} u(-\varepsilon, x)\right| \leq \begin{cases}M_{1} & j=0,1, \ldots,\lfloor\sigma\rfloor . \\ M_{1} \varepsilon^{\sigma-j} & j=\lfloor\sigma\rfloor+1, \ldots, m-1 .\end{cases}
$$

By the definition of $v$, we can take a constant $M_{2}>0$, by letting $R_{0}$ smaller if necessary, so that

$$
\left|\partial_{t}^{j} \partial_{x}^{\alpha} v(t, x)\right| \leq M_{2} \quad \text { in } \Delta\left(2 R_{0} ;-\varepsilon\right) \text { and for all }(j, \alpha) \in \Lambda,
$$

which implies

$$
\left|\left(\partial^{\Lambda} v(t, x)\right)^{\mu-\nu}\right| \leq M_{2}^{|\mu|-|\nu|} \quad \text { in } \Delta\left(2 R_{0} ;-\varepsilon\right) .
$$

This and Lemma 5.3 yield

$$
\left(\partial^{\Lambda} v(t, x)\right)^{\mu-\nu} \ll_{\varepsilon} C_{3} M_{2}^{|\mu|-|\nu|} \varphi_{\rho, R, \varepsilon}^{(0)}(t, x) .
$$

Using (5.12) and (5.19) we have

$$
f_{\mu}(t, x)\left(\begin{array}{c}
\mu \\
\nu
\end{array}\right)\left(\partial^{\Lambda} v(t, x)\right)^{\mu-\nu} \ll_{\varepsilon} C_{3} C_{5} A_{L}\left(\begin{array}{c}
\mu \\
\nu
\end{array}\right) L^{-|\mu|} M_{2}^{|\mu|-|\nu|} \rho^{-k_{\mu}} \varphi_{\rho, R, \varepsilon}^{(0)}(t, x) .
$$

We denote by $F_{\mu, \nu}(t, x)$ the right hand side of $(5.20)$ and consider the following majorant differential equation:

$$
\begin{gathered}
\partial_{t}^{m} W \gg_{\varepsilon} \sum_{\nu}\left(\sum_{\substack{\mu \in M \\
u \geq \nu}} F_{\mu, \nu}(t, x)\right)\left(\partial^{\Lambda} W\right)^{\Lambda}, \\
\partial_{t}^{J} W(-\varepsilon, x) \gg \begin{cases}0 & j=0,1, \ldots,\lfloor\sigma\rfloor, \\
\partial_{t}^{j} u(-\varepsilon, x) & j=\lfloor\sigma\rfloor+1, \ldots, m-1 .\end{cases}
\end{gathered}
$$

If $W$ satisfies the above majorant equation, then $w \ll_{\varepsilon} W$. In the same way as (5.15), putting

$$
W:=K \rho^{-\sigma} R^{m} \varphi_{\rho, R, \varepsilon}^{(m)}(t, x),
$$


we determine $K$ and $\rho$ so that $W$ satisfy (5.21a) with (5.21b).

Lemma 5.8. There is a constant $K_{1}>0$ such that $W$ defined by (5.22) with $K \geq K_{1} M_{1}$ satisfies the initial condition (5.21b). Here $K_{1}$ is independent of $\rho \geq 1$.

Proof. The proof is the same as that of Lemma 5.6.

Lemma 5.9. Let $L>K+M_{2}$. Then there is a constant $\rho_{1}=\rho_{1}(L) \geq 1$ such that $W$ defined by (5.22) with $\rho \geq \rho_{1}$ satisfies the majorant equation (5.21a).

Proof. We can prove the lemma in the same way as that of Lemma 5.7.

Now take $K \geq K_{1}, L>K+M_{2}$ and then $\rho \geq \rho_{1}$. Then $W$ defined by (5.22) satisfies the majorant equation (5.21a) and the initial condition $(5.21 \mathrm{~b})$, therefore we have $w \ll_{\varepsilon} W . W$ is holomorphic in $\left\{(t, x): \rho|t+\varepsilon|+\left|x_{1}\right|+\cdots+\left|x_{d}\right|\right.$ $<R\}$, which contains the origin of $\mathbb{C} \times \mathbf{C}^{d}$, and so is $w$. This shows $u=w+v$ is holomorphic near the origin of $\mathbf{C} \times \mathbb{C}^{d}$.

\section{References}

[1] Gérard, R. and Tahara. H., Holomorphic and singular solutions of nonlinear singular first order partial differential equations, Publ. RIMS, Kyoto Univ., 26 (1990), 979-1000.

[2] Solutions holomorphes et singulières d'équations aux dérivés partielles singulières non linéaires. Publ. RIMS, Kyoto Univ'.. 29 (1993), 121-151.

[3] Singular nonlinear partıal differential equations, Aspects of Mathematıcs, vol. E28, Vieweg, 1996.

[4] Ishii, T.. On propagation of regular singularities for nonlınear partial differential equations, $J$. Fac. Sci. Unvw. Tokyo, 37 (1990), 377-424.

[5] Kobayashi. T., On the prolongation of solutions for quasilinear differential equations, Proc. Japan Acad. Ser. A Math. Sci.. 60 (1984), no. 2, 60-62.

[6] Lax, P. D., Nonlinear hyperbolic equations, Comm. Pure Appl. Math., 6 (1953), 231-258.

[7] Leichtnam. É., Construction de solutions singulières pour des équations aux dérivées partielles non linéaires, Ann. Scı. École Norm. Sup. (4), 20 (1987), 137-170.

[8] Nabaji, A., Construction de solutions singuliéres pour des opérateurs quasi-lınéaires, Bull. Scr. Math., 119 (1995), no. 6, 509-527.

[9] Nabaji, A. and Wagschal, C., Singularités a croissance lente, J. Math. Pures Appl., 72 (1993), 335-375.

[10] Öuchi, S.. Formal solutions with Gevrey type estimates of nonlinear partial differential equations, J. Math. Sci. Univ. Tokyo, 1 (1994), 205-237.

[11] Tsuno, Y.. On the prolongation of local holomorphic solutions of nonlinear partial differential equations, J. Math. Soc. Japan. 27 (1975), 454-466.

[12] Wagschal, C., Le problème de Goursat non lınéaire. J. Math. Pures Appl., 58 (1979), 309-337.

[13] Weiss, J., The Painlevé property for partial differential equations. II: Bácklund transformation, Lax pairs, and the Schwarzian derivative, J. Math. Phys., 24 (1983). 1405-1413.

[14] Weiss, J., Tabor. M. and Carnevale, G., The Painlevé property for partial differential equations, J. Math. Phys., 24 (1983), 522-526.

[15] Zerner. M., Domaines, d'holomorphie des fonctions vérifiant une équation aux dérivées partielles. C. R. Acad. Sci. Paris Sér. I Math., 272(1971), 1646-1648. 
